\title{
Modulating the Faradic Operation of All-Printed Organic Electrochemical Transistors by Facile in Situ Modification of the Gate Electrode
}

\author{
Matteo Sensi, ${ }^{\dagger}$ Marcello Berto, ${ }^{\dagger}$ Andrea Candini, ${ }^{\ddagger}{ }^{\ddagger}$ Andrea Liscio, ${ }^{\ddagger}$, Andrea Cossarizza, $^{\prime \prime}$ \\ Valerio Beni, ${ }^{\perp}$ Fabio Biscarini, ${ }^{\dagger, \# \oplus}$ and Carlo Augusto Bortolotti ${ }^{*}{ }^{\dagger} \odot$ \\ ${ }^{\dagger}$ Dipartimento di Scienze della Vita, Università degli Studi di Modena e Reggio Emilia, Via Campi 103, 41125 Modena, Italy \\ ${ }^{\ddagger}$ Istituto per la Sintesi Organica e la Fotoreattività (ISOF)-CNR, Via P. Gobetti, 101, 40129 Bologna, Italy \\ ${ }^{\S}$ Istituto per la Microelettronica e Microsistemi (CNR-IMM), Via del Fosso del Cavaliere 100, 00133 Roma, Italy \\ "Dipartimento di Scienze Mediche e Chirurgiche Materno-Infantili e dell'Adulto, Università degli Studi di Modena e Reggio Emilia, \\ Via Campi 287, 41125 Modena, Italy \\ ${ }^{\perp}$ Department of Printed Electronics, RISE Acreo, Research Institute of Sweden, Norrköping 164 40, Sweden \\ \#Center for Translational Neurophysiology of Speech and Communication, Istituto Italiano di Tecnologia, Via Fossato di Mortara \\ 17-19, 44121 Ferrara, Italy
}

\section{Supporting Information}

\begin{abstract}
Organic electrochemical transistors (OECTs) operated in the faradic regime were shown as outperforming transducers of bioelectric signals in vitro and in vivo. Fabrication by additive manufacturing techniques fosters OECTs as ideal candidates for point-of-care applications, as well as imposes limitations on the choice of materials and their processing conditions. Here, we address the question of how the response of fully printed OECTs depends on gate electrode material. Toward this end, we investigate the redox processes underlying the operation of OECTs under faradic regime, to show OECTs with carbon gate (C-gate) that exhibit no current modulation gate voltages $<1.2 \mathrm{~V}$. This is a hallmark that no interference with the faradic operation of the device enabled by redox processes occurs when

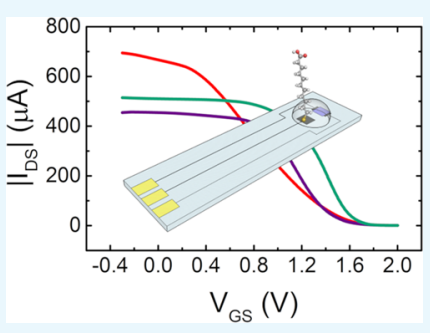
operating C-gate OECTs in the low-voltage range as label-free biosensors for the detection of electroactive (bio)molecules. To tune the faradic response of the device, we electrodeposited $\mathrm{Au}$ on the carbon gate (Au-C-gate), obtaining a device that operates at lower gate voltage values than C-gate OECT. The presence of gold on the gate allowed further modification of the electrical performances by functionalization of the $\mathrm{Au}-\mathrm{C}$-gate with different self-assembled monolayers by fast potential-pulseassisted method. Moreover, we show that the presence in the electrolyte solution of an external redox probe can be used to drive the faradic response of both $\mathrm{C}$ - and $\mathrm{Au}-\mathrm{C}$-gate $\mathrm{OECTs}$, impacting on the gate potential window that yields effective drain current modulation. The results presented here suggest possible new strategies for controlling the faradic operation regime of OECTs sensors by chemical modification of the gate surface.
\end{abstract}

\section{INTRODUCTION}

Electrolyte-gated organic transistors (EGOTs) are threeelectrode organic electronic devices in which an electrolyte solution acts as the gate dielectric ${ }^{1-6}$ in organic field effect transistors (OFETs), being ionically conductive and electronically insulating. ${ }^{7}$ Upon the gate voltage bias, ions accumulate at the interface between the organic semiconductor (OSC) and the electrolyte, else penetrate within the OSC channel. ${ }^{8,9}$ In the former case, the device behaves as electrolyte-gated organic field effect transistor (EGOFET), and in the latter case as organic electrochemical transistor (OECT). In both cases, the interaction between the ions and the active material controls the (de-)doping of the OSC channel and consequently the current flowing between source and drain $I_{\mathrm{DS}}$, hence the definition "electrolyte gating". ${ }^{10-12}$ The distinction between the two EGOT architectures is probably less marked than in the literature, as there are evidences of ion penetration in the
OSC of EGOFETs with substantial impact on the device response. ${ }^{9,13}$ The full elucidation of the molecular mechanisms underlying the operation of these devices is still subject of research.

The lack of a comprehensive theoretical framework has triggered the interest on EGOTs for applications in bioelectronics, neuroelectronics, neuromorphic computing, integration into logic circuits, and energy storage. EGOTs are attractive because of low-cost fabrication, lowpower operation, versatility, flexibility of device geometry and choice of materials, as well as biocompatibility.

To date, OECTs appear ahead of EGOFETs especially in in vivo transduction of bioelectric signals. ${ }^{3}$ Technologically,

Received: November 28, 2018

Accepted: March 5, 2019

Published: March 15, 2019 
OECTs rely mostly on poly(3,4-ethylenedioxy-thiophene) doped with polystyrene sulfonate (PEDOT:PSS) as the active material. PEDOT:PSS is commercially available as aqueous dispersion, and its ease of processing has boosted the demonstration of fully printed OECTs. ${ }^{7,16}$

Manufacturing chemo- and biosensors based on OECTs requires functionalization of the device interfaces with selective moieties toward the target analytes. In OECT printing, the functionalization of the channel is technologically challenging (unless adopting different strategies like electrodeposition of PEDOT, which is not easy to scale up). On one hand, EGOTs appear to be able to outperform state-of-the-art sensing solutions; on the other hand, their transfer to production is hindered by the lack of scalable and sustainable (in terms of time, cost, and manufacturing environmental conditions) strategies for tailoring interfaces and functional properties. ${ }^{17}$

Toward the aim to overcome these limitations, selfassembled monolayers (SAMs) represent an effective strategy for tailoring surfaces also of polycrystalline and amorphous technological substrates under not tightly controlled conditions (atmospheric pressure, absence of a clean room). ${ }^{18,19}$ SAMs were widely used in organic electronics to tune the charge injection barriers and control traps at the interface between channel and gate dielectric, but there are still a few examples of tailoring the gate electrode in EGOTs. ${ }^{18,20-22}$ One of the main drawbacks still hindering the widespread use of SAM-based modification is related to the time required to obtain a well-ordered, highly compact SAM, a process that typically requires hours, if not days, when performed at opencircuit potential (OCP).

For long-chain alkanethiols on Au, significant shortening of processing time can be achieved by a potential-controlled assembly, ${ }^{23,24}$ but a more time-effective strategy, which can be applied ideally to any SAM-forming thiol and even to biomacromolecules, is the potential-pulse-assisted method developed by Schuhmann and co-workers. ${ }^{25}$ This strategy, based on pulse-type potential modulation, yields SAM formation on polycrystalline gold within a few minutes and depends on the relationship between timestep and the length of the alkyl chain and on the point of zero charge of the electrode before and after SAM formation, which determines the potential range of the steps.

In this work, we demonstrate the modulation of the operation of fully printed OECTs by in situ, solution-based modification of the gate electrode in the minute timescale, under environmentally friendly conditions, through easily scalable procedures. The pristine devices feature a carbon gate, and we sequentially modify them first by the electrodeposition of a gold layer and then by the potential-pulseassisted formation of two different SAMs on the electrodeposited $\mathrm{Au}$. We investigate to what extent the modification of the gate electrodes affects the working mechanism of the devices and consequently the electrical performances, thus defining their potential application range for different technological purposes.

\section{RESULTS AND DISCUSSION}

A schematic of the screen-printed OECTs used in the present work is provided in Figure 1a. Source, drain, and gate electrodes are all made of carbon, and PEDOT:PSS was deposited as channel material. The gate electrode and the channel feature the same area. PEDOT:PSS-based OECTs work in depletion mode, meaning that they are in the ON state
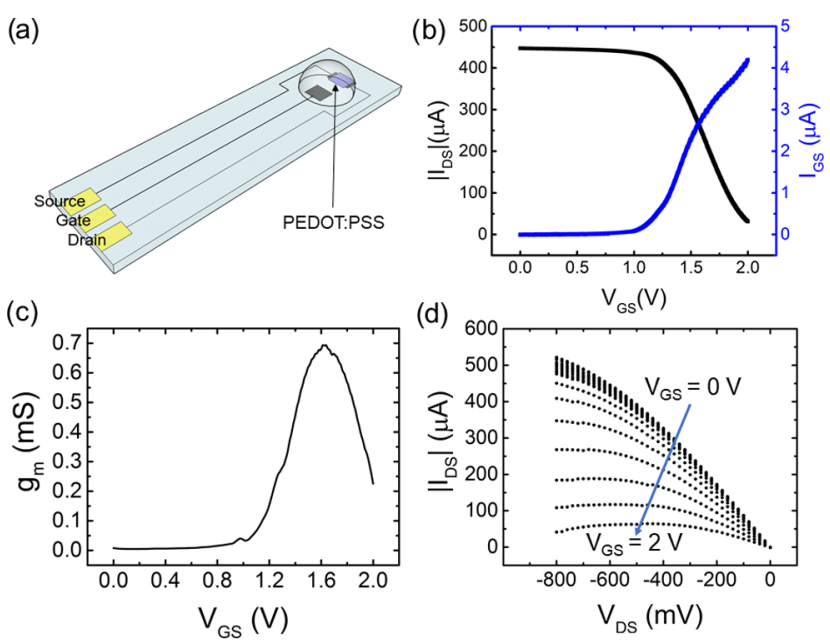

Figure 1. OECT with carbon gate (C-gate). (a) Schematic of the device. (b) Transfer characteristics and (c) transconductance of the device; $V_{\mathrm{DS}}$ was fixed at $-0.4 \mathrm{~V}$. (d) Output curves, recorded with a $V_{\mathrm{GS}}$ step of $0.2 \mathrm{~V}$. All measurements were performed in phosphatebuffered saline (PBS) $50 \mathrm{mM}, \mathrm{pH} 7.4$.

when no $V_{\mathrm{GS}}$ is applied, and a hole current is established by applying a voltage between the source and drain electrodes $\left(V_{\mathrm{DS}}\right)$; the application of a positive $V_{\mathrm{GS}}$ leads to polymer dedoping, with the current between drain and source $\left(I_{\mathrm{DS}}\right)$ decreasing and the device entering the OFF state.

OECTs can work under two different regimes. ${ }^{11,26,27}$ The nonfaradic regime exploits capacitive charging of an ionic double layer at the gate electrode, which results in ions of opposite sign entering the channel and (de)doping the conducting polymer by compensation of the sulfonate anions. In the faradic regime, channel (de)doping is caused by faradic processes (i.e., reduction or oxidation) at the gate electrode. The choice of the gate material (i.e., how closely it behaves as an ideally polarizable electrode, and its electroactivity), of the electrolyte solution, and, clearly, also of the applied $V_{\mathrm{GS}}$ range defines whether the OECT response will be dominated by nonfaradic or faradic operation. Faradic operation is typically neglected when the gate current $\left(I_{\mathrm{GS}}\right)$ is far lower $(<1 \%)$ than those recorded at the drain $\left(I_{\mathrm{DS}}\right)$; this might lead to inconsistencies, as it neglects the intrinsic amplification provided by the OECT working mechanism to faradic processes happening at the gate.

The transfer characteristic of an all-printed carbon gate OECT (C-gate OECT hereafter), recorded in PBS $50 \mathrm{mM}$ $(\mathrm{pH} 7.4)$, is reported in Figure $1 \mathrm{~b}$ and is in agreement with the expected depletion mode operation. Both $I_{\mathrm{DS}}$ and $I_{\mathrm{GS}}$, recorded at $V_{\mathrm{DS}}-0.4 \mathrm{~V}$, are shown and a rapid decrease of $I_{\mathrm{DS}}$ can be observed only when oxidation processes (in this specific case, water oxidation) take place at the carbon gate electrode, as mirrored by the steep increase in $I_{\mathrm{GS}}$ for $V_{\mathrm{GS}} \geq 1.2 \mathrm{~V}$. This is further confirmed by the plot of transconductance $g_{\mathrm{m}}$ vs $V_{\mathrm{GS}}$ (Figure 1c), with $g_{\mathrm{m}}$ defined as follows

$$
g_{\mathrm{m}}=\partial I_{\mathrm{DS}} / \partial V_{\mathrm{GS}}
$$

In the present case, $g_{\mathrm{m}}$ takes non-null values in the same range as above with onset at $V_{\mathrm{GS}}=1.0 \mathrm{~V}$, reaching a maximum of about $0.7 \mathrm{mS}$ at $V_{\mathrm{GS}} \sim 1.6 \mathrm{~V}$. The observed behavior is in line with both the poor polarizability and the large window of electrochemical stability (i.e., absence of redox processes 
(a)

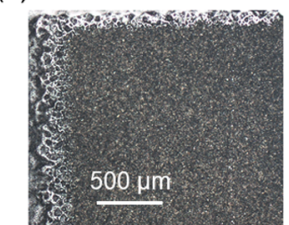

C-gate

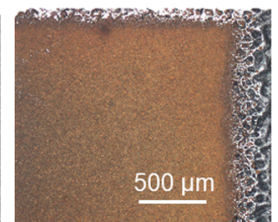

Au-C-gate

(c)

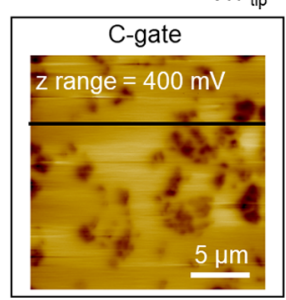

$W F_{\text {tip }}-W F_{\text {sample }}$

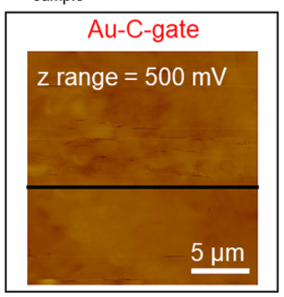

(b)

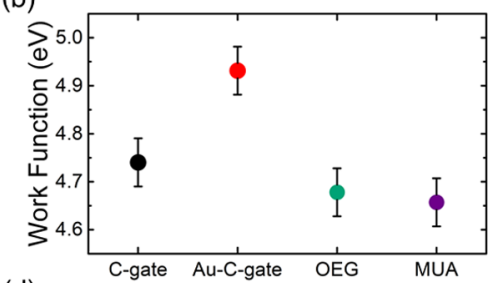

(d)

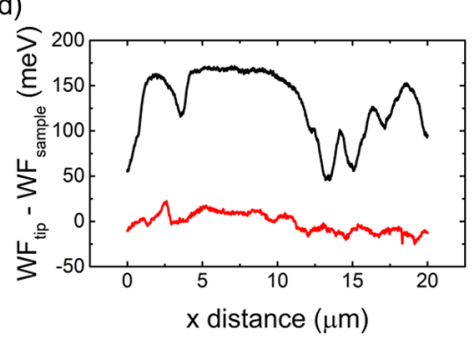

Figure 2. Au-C-gate characterization by optical microscopy and KPFM. (a) Optical microscopy picture of the gate surface before (left) and after (right) gold electrodeposition. (b) Work function of the C-gate, Au-C-gate, and Au-C-gate OECTs functionalized with SAMs. (c) Difference between the work function of the tip and the work function of the sample and (d) its profile for C-gate and Au-C-gate OECTs.

involving the gate electrode material and/or the electrolyte solution) at the printed carbon electrode.

As a result of the low polarizability of carbon electrode, the OECT does not show nonfaradic operation, and de-doping can be observed only when water oxidation takes place at the gate; this process must be coupled to a half-reaction, which most likely involves PEDOT reduction, according to the well-known reaction

$$
\text { PEDOT }^{+}: \text {PSS }^{-}+\mathrm{Y}^{+}+\mathrm{e}^{-} \rightleftharpoons \mathrm{PEDOT}+\mathrm{Y}^{+}: \mathrm{PSS}^{-}
$$

where $\mathrm{Y}^{+}$represents a generic cation that from the water solution (left-hand side) enters the polymer blend and binds to PSS. $^{27}$ In the faradic regime of operation for PEDOT:PSS OECTs, modulation is achieved at gate potential values that yield oxidation processes at the gate and reduction of PEDOT in the channel, as from eq 2. Thus, a possible oxidation semireaction that complements eq 2 is

$$
\frac{3}{2} \mathrm{H}_{2} \mathrm{O} \rightarrow \frac{1}{4} \mathrm{O}_{2}+\mathrm{e}^{-}+2 \mathrm{H}_{3} \mathrm{O}^{+}
$$

In the case of C-gate OECTs, the output characteristics confirm that modulation is achieved only at $V_{\mathrm{GS}}$ values that allow water oxidation.

To obtain the output curves, the drain was negatively biased and swept between 0 and $-0.8 \mathrm{~V}$, with $V_{\mathrm{GS}}$ ranging from 0 to $+2 \mathrm{~V}$, with a $+0.2 \mathrm{~V}$ step. The transition from a linear to a saturation regime, with an almost flat saturation current, can be observed for $V_{\mathrm{GS}} \geq 1.2 \mathrm{~V}$. The absence of effective modulation in the $V_{\mathrm{GS}}$ range of $0-1 \mathrm{~V}$, coinciding with the stability window of water, makes carbon gate OECTs an ideal platform for the development of (i) sensors based on enzymatic reactions (e.g., first-generation oxidase-based biosensors ${ }^{28}$ ), (ii) sensors for the detection of electroactive biomolecules as dopamine $^{29}$ or ascorbic acid, and (iii) ideal substrate for the immobilization of a variety of inorganic catalysts for the enzymeless detection of molecules of biological relevance (e.g., glucose).$^{30}$ At the same time, it shows that a carbon gate electrode is not ideal when low-voltage $(\ll 1 \mathrm{~V})$ modulation is desired or when the OECT has to amplify a potential variation within the water stability window into a current change.
The use of gold instead of carbon as the gate electrode material represents a viable alternative: $\mathrm{Au}$ gates in OECTs are known to ensure effective modulation ${ }^{31}$ and a large arsenal of protocols for Au modification, tailoring the sensing ability (e.g., biomolecule immobilization) and overall properties, is widely reported in the literature.

Printing $\mathrm{Au}$ on poly(ethylene terephthalate) (PET) requires high cost and temperature in the processing. One possibility to maintain cost-effectiveness and fabrication on a flexible device is to electrodeposit a gold layer on top of the printed carbon gate. To achieve this goal, we followed a well-established protocol for $\mathrm{Au}$ electrodeposition (10 $\mathrm{min}$ by potentiostatic control at $-0.273 \mathrm{~V}$ vs $\mathrm{Ag} / \mathrm{AgCl}$ in $3 \mathrm{mM} \mathrm{HAuCl}_{4}, 0.1 \mathrm{M}$ $\mathrm{KCl}^{32}$ ), which yields an environmentally friendly surface modification in aqueous solutions and at room temperature. The fabrication of $\mathrm{Au}-\mathrm{C}$-gate electrodes, featuring the presence of $\mathrm{Au}$ layer on top the carbon electrode, was monitored by both optical microscopy (Figure 2a) and Kelvin probe force microscopy (KPFM) measurements. The work function (WF) of the gate interfaces, as measured by KPFM, increases from $\sim 4.7$ to $\sim 4.9 \mathrm{eV}$ upon gold electrodeposition on carbon, the latter value being in line with published work function values for polycrystalline $\mathrm{Au} .{ }^{33}$ Moreover, KPFM also enables the mapping of the potential spatial distribution with nanoscale resolution, thus providing information on how (in)homogeneous is the gate morphology. As evidenced in Figure $2 \mathrm{c}, \mathrm{d}$, after the $\mathrm{Au}$ deposition, the gate surface potential (SP) is more uniform, although still highly influenced by the morphologically heterogeneous carbon electrode underneath.

The electrical response of the $\mathrm{Au}-\mathrm{C}$-gate OECTs (red curves in Figure $3 a-d$ ) shows a marked improvement with respect to the pristine one (black curves in the same figure panels): the devices exhibit proper transistor response, and $V_{\mathrm{GS}}$ modulation is much more effective in the low potential $\left(V_{\mathrm{GS}}<\right.$ $1 \mathrm{~V}$ ) window, with $g_{\mathrm{m}}$ reaching its maximum value of about $0.65 \mathrm{mS}$ at $V_{\mathrm{GS}} \sim 0.8 \mathrm{~V}$. The change in the electrode material also affects the drain current value at low voltages, which for the $\mathrm{Au}-\mathrm{C}$-gate OECT is about $40 \%$ higher than that of the Cgate one. The current difference can be ascribed to different active areas of the gate electrodes and to the larger impedance of carbon compared to gold, also considering that in the 

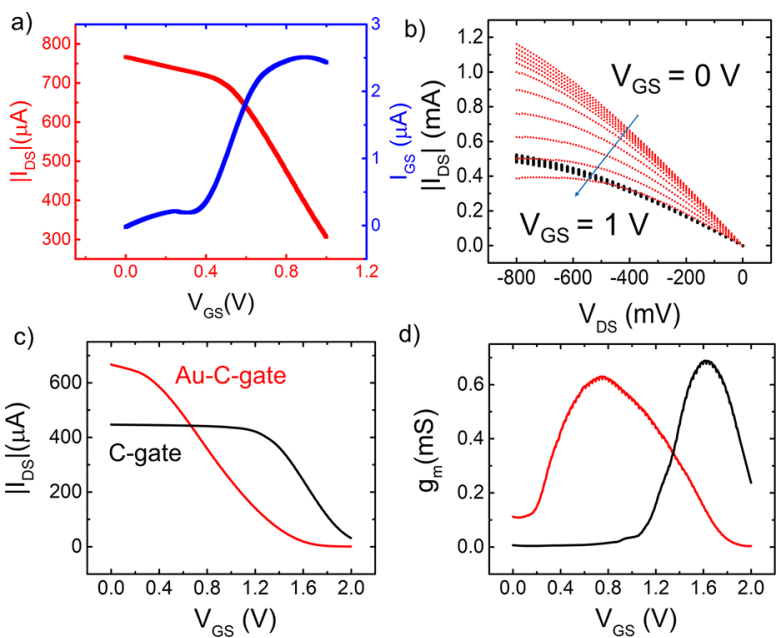

Figure 3. Au-C-gate OECT. (a) Transfer characteristics of the device, $V_{\mathrm{DS}}$ was fixed at $-0.4 \mathrm{~V}$. (b) Comparison between the output and $(\mathrm{c})$ transfer $\left(V_{\mathrm{DS}}=-0.4 \mathrm{~V}\right)$ curves of the devices with C-gate and $\mathrm{Au}-\mathrm{C}$-gate. (d) Comparison of the transconductance obtained from the curves in (c). All measurements were performed in PBS $50 \mathrm{mM}$, $\mathrm{pH}$ 7.4.

present case, the gate is composed of a printable ink, i.e., a dispersion of nanometer-sized carbon particles.

The comparison of $I_{\mathrm{DS}}$ and $I_{\mathrm{GS}}$ in Figure 3a suggests a faradic regime of operation also for the $\mathrm{Au}-\mathrm{C}$-gate devices, but, at variance with what was observed for C-gate OECTs, here the faradic gate current, peaking at $\sim 0.8 \mathrm{~V}$, can be ascribed to $\mathrm{Au}$ oxidation, ${ }^{34}$ whereas water oxidation would occur at more positive potential values.

Both C-gate and $\mathrm{Au}-\mathrm{C}$-gate $\mathrm{OECTs}$ exhibit high stability, as witnessed by the transfer curves recorded after continuous cycling $(6 \mathrm{~h})$ and 2 days of storage in air (see Figure S2 in the Supporting Information (SI)). The reproducibility of the Cgate and $\mathrm{Au}-\mathrm{C}$-gate $\mathrm{OECT}$ performances was also investigated, by comparing the transfer curves obtained from eight distinct devices. Interestingly, we observe a decrease of the standard deviation of the transfer characteristics after gold electrodeposition.

The adoption of Au layer allows us to further modify the gate electronic properties by the deposition of SAM. Here, to demonstrate this capability, we show the functionalization with alkanethiols SAMs. This choice was dictated by the fact that the thiol/Au interaction is well documented, in terms of both functionalization strategies and understanding, and because alkanethiol self-assembly has been vastly explored for the immobilization on $\mathrm{Au}$ surfaces of biomolecules (e.g., proteins, oligonucleotides), also for sensing application. ${ }^{20,22,35,36}$

Here, we modified the $\mathrm{Au}-\mathrm{C}$-gate OECTs through the potential-assisted deposition $^{25,37}$ of two different SAMs: 11mercaptoundecanoic acid (MUA) and 11-mercaptoundecyltriethylene glycol (OEG). The potential-pulse-assisted deposition of SAMs on the gate of the OECT was performed in situ, i.e., in the exact same setup used for the previously described electrical characterization. Such setup includes measurement performed in a Faraday cage, by means of a source meter unit and upon confinement of the mixed aqueous/alcoholic SAM solution on the OECT gate and channel with a poly(dimethylsiloxane) (PDMS) pool. All modifications and characteristics were performed at room temperature. $V_{\mathrm{GS}}$ was pulsed between 0 and $0.5 \mathrm{~V}$ with a timestep of $3 \mathrm{~s}$ for a total time of 3 and 6 min for OEG and MUA, respectively, and the drain current was monitored during the functionalization procedure upon application of a fixed $V_{\mathrm{DS}}(-0.4 \mathrm{~V}$, see Figures S6 and S7 in the SI). The formation of the monolayer was confirmed by recording cyclic voltammograms (CVs) in $5 \mathrm{mM}$ $\mathrm{K}_{3}\left[\mathrm{Fe}(\mathrm{CN})_{6}\right]^{3-}$ and $0.1 \mathrm{M} \mathrm{KCl}$, using a classic three-electrode system, where the OECT gate was connected as the working electrode (see Materials and Methods). The comparison of the cyclic voltammograms recorded for bare $\mathrm{Au}-\mathrm{C}$-gate electrodes with those obtained after the SAM formation is depicted in Figure 4. For both SAMs, the potential-pulse-assisted
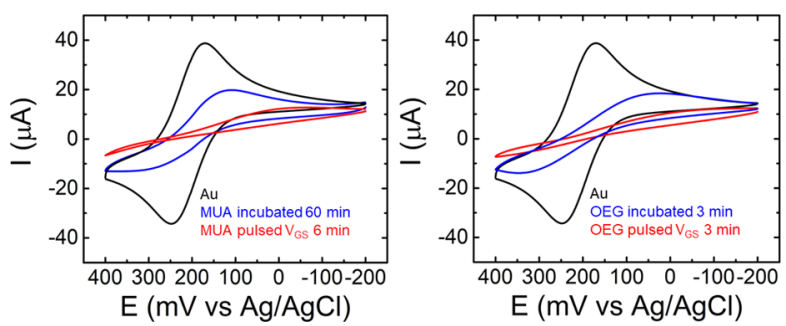

Figure 4. $\mathrm{CV}$ of the $\mathrm{Au}-\mathrm{C}$-gate $\mathrm{OECT}$ functionalized with MUA (left) or OEG (right) by incubation (blue trace) or by potentialpulse-assisted method (red trace). The CVs were recorded in $5 \mathrm{mM}$ $\mathrm{K}_{3}\left[\mathrm{Fe}(\mathrm{CN})_{6}\right], 0.1 \mathrm{M} \mathrm{KCl}$.

functionalization leads to a nearly complete passivation of the surface within minutes, as witnessed by the marked decrease of both anodic and cathodic peak currents, suggesting prevented access of the redox probe to the gold surface. The potential-pulse-assisted procedure was compared with immobilization performed via incubation at OCP in ethanolic solution of the two thiols: the corresponding CVs, also shown in Figure 4, indicate that lower coverage (49\% for MUA and $52 \%$ for OEG, see Materials and Methods) by SAM formation is achieved in the absence of $V_{\mathrm{GS}}$ pulses, despite longer (for MUA, since no coverage is observed in $6 \mathrm{~min}$ ) or identical (for OEG) incubation time. KPFM provides indirect proof of the presence of the SAMs on the gate surface: the work function for both OEG- and MUA-modified gate shifts to lower values (4.68 and 4.66 for OEG-Au-C-gate and MUA-Au-C-gate electrodes, respectively; see Figure $2 \mathrm{~b}$ ), in line with literature values. $^{38}$

We then investigated how the MUA and OEG functionalization affects the OECT performances. The first observation is that the devices can withstand exposure to a 1:1 water/ethanol solution and still be able to work properly afterward (see Figure S5 in the Supporting Information).

The transfer characteristics, obtained by $V_{\mathrm{GS}}$ sweeping in the $0-2 \mathrm{~V}$ range, show that, despite reaching lower $I_{\mathrm{DS}}$ values with respect to $\mathrm{Au}-\mathrm{C}$-gate $\mathrm{OECT}$ s, both SAM-modified devices still provide proper current modulation. Inspection of the gate currents (Figure $5 \mathrm{~b}$ ) suggests that both $\mathrm{OEG}-\mathrm{Au}-\mathrm{C}$-gate and $\mathrm{MUA}-\mathrm{Au}-\mathrm{C}$-gate OECTs work under the faradic regime of operation: it is reasonable to infer that the faradic process at the gate that drives the PEDOT reduction and channel dedoping is still the oxidation of the Au surface. Nevertheless, the presence of the SAM on the electrode surface is hindering the metal oxidation pushing the required potential toward more positive values, ${ }^{39}$ as also witnessed by the transconductance trend, with $g_{\mathrm{m}}$ maxima of $0.8 \mathrm{mS}$ at about $1.1 \mathrm{~V}$ and $1 \mathrm{mS}$ at $1.5 \mathrm{~V}$ for MUA and OEG, respectively. Modulation occurring at less positive potentials for MUA-Au-C-gate OECTs with 


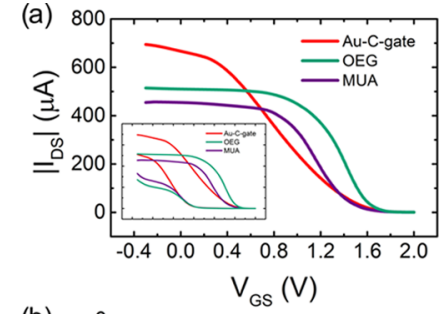

(b)

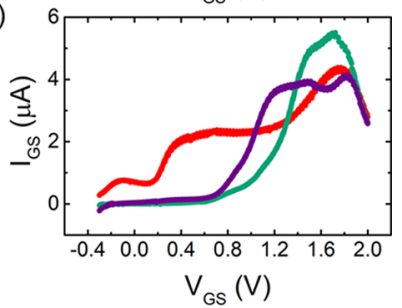

(c)

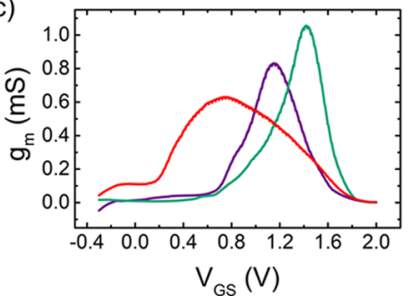

Figure 5. Transfer characteristics in the presence of SAMs. (a) Transfer curves and (b) gate current of the OECT with $\mathrm{Au}-\mathrm{C}$-gate and after functionalization with MUA or OEG, $V_{\mathrm{DS}}=-0.4 \mathrm{~V}$. Complete transfer curves are reported in the inset of (a). (c) Transconductance calculated from the curves in (a). All measurements were performed in PBS $50 \mathrm{mM}, \mathrm{pH} 7.4$.

respect to $\mathrm{OEG}-\mathrm{Au}-\mathrm{C}$-gate ones can be ascribed to differences in the compactness and stability of OEG and MUA SAMs. In particular, OEG modification is expected to yield a more compact layer, while the electrostatic repulsion occurring between the carboxylic headgroups in MUA should affect the monolayer stability, with repercussions on the overpotential required for $\mathrm{Au}$ oxidation and, subsequently, on the OECT modulation. By performing a series of control experiments, we confirmed that the figures of merit for SAMbased devices were not to be ascribed to neither exposure to the mixed organic/aqueous solution nor the application of the voltage pulses alone, but rather to the formation of the monolayer on the gate (see SI Figures S5-S7).

One can improve the faradic modulation of OECTs by exploiting the electrochemical activity of a reversible redox couple intentionally added to the system either by immobilization on the gate ${ }^{26}$ or by adding it to the electrochemical gating solution. ${ }^{40}$ To demonstrate this possibility and to highlight its advantages in modulating OECTs response, we compared the transfer curves obtained with the $\mathrm{C}$-gate and the $\mathrm{Au}-\mathrm{C}$-gate OECTs (Figure 6a,b) in $50 \mathrm{mM}$ PBS in the presence (red curves) and absence (black curves) of $\mathrm{K}_{3}\left[\mathrm{Fe}(\mathrm{CN})_{6}\right]$ at $5 \mathrm{mM}$ concentration. In the presence of the redox couple, drain current modulation under faradic regime takes place at less positive $V_{\mathrm{GS}}$ values compared to those needed for the water oxidation at $\mathrm{C}$-gate and gold oxidation at $\mathrm{Au}-\mathrm{C}$-gate devices. Moreover, the hysteresis is dramatically reduced due to the reversibility of the ferro/ferricyanide redox couple compared to the $\mathrm{Au} / \mathrm{AuOx}$ redox couple (Figure $6 \mathrm{~b}$ ) and to the absence of reduction reaction at $\mathrm{C}$-gate in the investigated potential window.
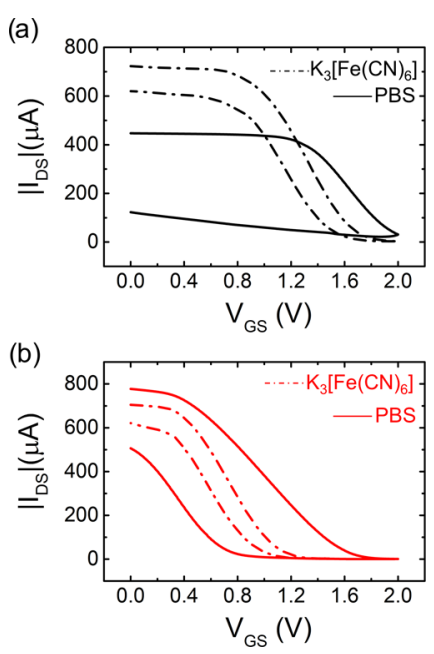

Figure 6. Transfer cycles in PBS $50 \mathrm{mM}$ (solid line) and in $5 \mathrm{mM}$ $\mathrm{K}_{3}\left[\mathrm{Fe}(\mathrm{CN})_{6}\right], 0.1 \mathrm{M}$ PBS (point dashed line) of (a) C-gate and (b) $\mathrm{Au}-\mathrm{C}$-gate OECTs; the $V_{\mathrm{DS}}$ was fixed at $-0.4 \mathrm{~V}$.

\section{CONCLUSIONS}

In this paper, we reported on the effect of the gate material and its functionalization on the electrical performances of OECTs. We described facile modification of the gate electrode via potentially scalable strategies that lead to modulation of the OECTs operation regime. We observe that $\mathrm{Au}$ electrodeposition leads to an overall improvement of the device performances in comparison to pristine C-gate OECTs, ensuring higher current and, most importantly, low-voltage modulation. Despite still working under faradic operation regime, the oxidation process at the gate is different for $\mathrm{Au}-\mathrm{C}$ gate OECTs and takes place at less positive $V_{\mathrm{GS}}$ values than in C-gate. The potential window, in which the printed OECTs exhibit drain current modulation, can be further tuned and narrowed by exploiting reversible oxidation/reduction of potassium ferro-ferricyanide system at the gate electrode.

Rapid functionalization via potential-pulse-assisted SAM formation has been exploited here to tailor the electrical performances of OECTs. We tested two SAM-forming molecules that differ with respect to the expected lateral interactions upon formation of the layer, impacting on the compactness of the latter. With MUA SAMs, the carboxylic headgroup undergoes (de)protonation equilibria that make it negatively charged under physiological conditions. We infer that the use of other SAMs, varying in terms of chain length, chemical properties of the headgroup, and packing, will enable fine tuning of the OECT performances, also by providing tethers for further functionalization of the gate with molecules serving as recognition moieties or switches. ${ }^{41}$

The faradic regime for OECTs is characterized by sensitivity of $I_{\mathrm{DS}}$ to $V_{\mathrm{GS}}$ larger than in nonfaradic regime, since in the former case, the potential drop is mostly taking place at the electrolyte/channel interface, while the solution potential $V_{\text {sol }}$ is nearly equal to the potential of the gate $V_{\mathrm{GS}}$. This paper provides deeper insights into the molecular events underlying $I_{\mathrm{DS}}$ modulation by redox processes with varying gate materials.

The reported results open two potential scenarios: (i) the use of redox molecules could be envisaged as an effective way of monitoring interfacial reactions (e.g., biorecognitions in affinity or immuno-sensors) and (ii) C-gate and gold oxidation at $\mathrm{Au}-\mathrm{C}$-gate devices could serve as sensing platform for 
molecules (e.g., dopamine) or process (e.g., enzymatic reaction) having or generating products with intrinsic redox activity. We believe that modulation of the faradic operational regime in fully printed OECTs with low-cost approaches will provide further routes to the exploitation of OECTs and especially in (bio) sensing applications.

\section{MATERIALS AND METHODS}

Device Fabrication. The OECT was manufactured by screen-printing on PET (Polyfoil Bias; thickness $=125 \mu \mathrm{m}$ ). A commercial Ag ink (Ag5000, DuPont, U.K.) was used to print the conductive tracks; the carbon gate and the carbon contact pads were printed using a commercial carbon ink (C2130307D1, Gwent, U.K.), while the channel of the device was obtained by printing a PEDOT:PSS commercial ink (Clevios SV3, Heraeus Group, Germany). By coating with a dielectric layer (5018, DuPont, U.K.), we left exposed only the gate, the channel, and the final contacts of the conductive tracks. All of the devices used in this work have the geometry described in the following paragraph. The area of the gate is 4 $\mathrm{mm}^{2}$, while the channel has a dimension of $2 \times 3 \mathrm{~mm}^{2}$. The channel thickness, as determined by atomic force microscopy, was $280 \pm 20 \mathrm{~nm}$. A PET gap of $4 \mathrm{~mm}^{2}$ is placed between the two components. The OECT was printed by using a semiautomatic screen printer (DEK Horizon 03i printer, ASM Assembly Systems GmbH, Germany).

We determined an active area of the C-gate of $\sim 1.9 \mathrm{~mm}^{2}$, by applying the Randles-Sevçik equation to CVs measured in 0.1 $\mathrm{M} \mathrm{KCl}$ and $5 \mathrm{mM} \mathrm{K}_{3}\left[\mathrm{Fe}(\mathrm{CN})_{6}\right]$ at $100 \mathrm{mV} \mathrm{s}^{-1}$.

To electrodeposit gold on the carbon gate of the device, we used a $\mathrm{CH}$ Instruments potentiostat $760 \mathrm{c}$ model with a threeelectrode setup, where the gate was connected as the working electrode alongside an $\mathrm{Ag} / \mathrm{AgCl}$ reference electrode (ElbaTech, Livorno, Italy) and a Pt wire as the counter electrode, all in contact with the plating aqueous solution confined by a PDMS pool. Gold was electrodeposited on the carbon gate of the device in $10 \mathrm{~min}$ by performing chronoamperometry at $-0.273 \mathrm{~V}$ vs $\mathrm{Ag} / \mathrm{AgCl}$ in $3 \mathrm{mM} \mathrm{HAuCl}_{4}$ (commercial gold plating solution: $50 \mathrm{~mL}$ of $24 \mathrm{k}$ gold plating solution) and 0.1 $\mathrm{M} \mathrm{KCl}$. Reproducibility of the printing and plating process was tested by performing transfer experiments with eight independent OECTs (Figure S2e,f).

Gate Functionalization. To functionalize the gate, we used two SAMs: 11-mercaptoundecyl-triethylene glycol (OEG), $0.1 \mathrm{mM}$ PBS (50 mM, pH 7.4), or 11-mercaptoundecanoic acid (MUA), $1 \mathrm{mM}$ in a mixed water and ethanol solution, in a $1: 1$ ratio, with $10 \mathrm{mM} \mathrm{NaCl}$. The deposition of the SAM was obtained at room temperature either by the potential-pulse-assisted procedure or by incubating the device in a $2 \mathrm{~mL}$ tube filled with the SAM solution for $3 \mathrm{~min}$ (OEG) or $60 \mathrm{~min}$ (MUA). The choice of a different timescale of incubation for the two SAMs is due to the faster self-assembly of OEG compared to MUA. For the potential-pulse-assisted procedure, we confined the OEG or MUA solution with a PDMS pool and applied $V_{\mathrm{GS}}$ steps $(0$ and $0.5 \mathrm{~V})$ in a current vs time measurement, with or without applying a $V_{\mathrm{DS}}$. At the end of the measurement, we removed the solution and rinsed with PBS.

Electrochemical Characterization. We recorded cyclic voltammograms (CVs) to verify and estimate the gate surface coverage upon functionalization with the SAMs (see Figure 4) by applying the Randles-Sevçik equation.
The setup for the electrochemical characterization is the same described in the previous section. The CVs were performed in $0.1 \mathrm{M} \mathrm{KCl}$ and $5 \mathrm{mM} \mathrm{K}_{3}\left[\mathrm{Fe}(\mathrm{CN})_{6}\right]$ at 100 $\mathrm{mV} \mathrm{s}^{-1}$ to monitor the cathodic peak corresponding to the reduction of potassium ferricyanide.

Electrical Characterization. The electrical characteristics were performed at room temperature by connecting the source, drain, and gate electrodes to an Agilent B2902A Source Meter Unit and by confining a PBS solution $(50 \mathrm{mM}, \mathrm{pH} 7.4)$ or a $0.1 \mathrm{M}$ PBS and $5 \mathrm{mM} \mathrm{K}_{3}\left[\mathrm{Fe}(\mathrm{CN})_{6}\right]$ solution in a PDMS pool. To record $I-V$ transfer curves, the drain-source voltage $\left(V_{\mathrm{DS}}\right)$ was fixed at $-0.4 \mathrm{~V}$, while the gate-source voltage $\left(V_{\mathrm{GS}}\right)$ was swept from 0 to 1 or $2 \mathrm{~V}$. The output characteristics were obtained by sweeping both $V_{\mathrm{GS}}$, from 0 to 1 or $2 \mathrm{~V}$, and $V_{\mathrm{DS}}$, from 0 to $-0.8 \mathrm{~V}$.

Atomic Force Microscopy and Kelvin Probe Force Microscopy. Atomic force microscopy (AFM) and Kelvin probe force microscopy (KPFM) measurements were performed with a commercial microscope Multimode 8 (Bruker) operated in air, employing Pt/Ir-coated cantilever doped silicon tips (SCM-PIT-V2, Bruker), with mechanical constant $k=3 \mathrm{~N} / \mathrm{m}$ and oscillating frequency $f_{0} \sim 75 \mathrm{kHz}$. AFM and KPFM images were acquired in the same measurement: first, a topographic line scan is performed in the tapping mode, second, the same line is rescanned at a lift height of $50 \mathrm{~nm}$, using the amplitude modulation mode. The KPFM technique provides a voltage resolution of about $5 \mathrm{mV}$ and a lateral resolution of a few tens of nanometers. Raw AFM and KFPM data were treated by SPIP software (https://www. imagemet.com/products/spip/) using flattening procedures to correct for the piezo-scanner-induced artifacts. ${ }^{42}$ Comprehensive description of the two techniques can be found in refs ${ }^{43}$ and 44 and references therein.

For each of the analyzed sample, the work function WF was estimated by taking the average surface potential SP value obtained on several $20 \times 20 \mu \mathrm{m}^{2}$ images in different regions of the sample and subtracting it from the value of the tip WF, calibrated onto freshly cleaved HOPG $\left(\mathrm{WF}_{\mathrm{HOPG}}=4.65 \mathrm{eV}\right) .^{45}$

\section{ASSOCIATED CONTENT}

\section{Supporting Information}

The Supporting Information is available free of charge on the ACS Publications website at DOI: 10.1021/acsomega.8b03319.

Point of zero charge determination; assessment of stability of the devices under different conditions (varying solvent, continuous cycling, prolonged storage in air); plot of drain-source current during potentialpulse-assisted functionalization with OEG and MUA; investigation of the impact of the gate area on the electrical properties of devices; and demonstration of the use of C-gate OECT for detection of ascorbic acid (PDF)

\section{AUTHOR INFORMATION}

\section{Corresponding Author}

*E-mail: carloaugusto.bortolotti@unimore.it.

ORCID $\odot$

Matteo Sensi: 0000-0002-0491-7161

Marcello Berto: 0000-0002-3356-8829

Andrea Candini: 0000-0003-3909-473X

Fabio Biscarini: 0000-0001-6648-5803 
Carlo Augusto Bortolotti: 0000-0002-5701-5727

Notes

The authors declare no competing financial interest.

\section{ACKNOWLEDGMENTS}

This work was funded by EuroNanoMed III project "AMI”, by the Life Science Department of the University of Modena and Reggio Emilia, through "FAR2017", and by "Fondazione di Vignola". It was also funded by the European Commission Horizon 2020 research and innovation programme under "GrapheneCore2 785219-Graphene Flagship" and the Swedish Foundation for Strategic Research (Smart Intrabody network; grant RIT15-0119). The authors thank Marie Nilsson and Chiara Diacci for OECT fabrication and Simone Benaglia for the channel thickness measurement by AFM.

\section{REFERENCES}

(1) Kergoat, L.; Herlogsson, L.; Braga, D.; Piro, B.; Pham, M. C.; Crispin, X.; Berggren, M.; Horowitz, G. A Water-Gate Organic FieldEffect Transistor. Adv. Mater. 2010, 22, 2565-2569.

(2) Wang, D.; Noël, V.; Piro, B. Electrolytic Gated Organic FieldEffect Transistors for Application in Biosensors-A Review. Electronics 2016, 5, 9.

(3) Rivnay, J.; Inal, S.; Salleo, A.; Owens, R. M.; Berggren, M.; Malliaras, G. G. Organic Electrochemical Transistors. Nat. Rev. Mater. 2018, 3, No. 17086.

(4) Melzer, K.; Brändlein, M.; Popescu, B.; Popescu, D.; Lugli, P.; Scarpa, G. Characterization and Simulation of Electrolyte-Gated Organic Field-Effect Transistors. Faraday Discuss. 2014, 174, 399411.

(5) Toss, H.; Suspène, C.; Piro, B.; Yassar, A.; Crispin, X.; Kergoat, L.; Pham, M. C.; Berggren, M. On the Mode of Operation in Electrolyte-Gated Thin Film Transistors Based on Different Substituted Polythiophenes. Org. Electron. 2014, 15, 2420-2427.

(6) Torsi, L.; Magliulo, M.; Manoli, K.; Palazzo, G. Organic FieldEffect Transistor Sensors: A Tutorial Review. Chem. Soc. Rev. 2013, $42,8612$.

(7) Kim, S. H.; Hong, K.; Xie, W.; Lee, K. H.; Zhang, S.; Lodge, T. P.; Frisbie, C. D. Electrolyte-Gated Transistors for Organic and Printed Electronics. Adv. Mater. 2013, 25, 1822-1846.

(8) Tarabella, G.; Mahvash Mohammadi, F.; Coppedè, N.; Barbero, F.; Iannotta, S.; Santato, C.; Cicoira, F. New Opportunities for Organic Electronics and Bioelectronics: Ions in Action. Chem. Sci. 2013, 4, 1395-1409.

(9) Di Lauro, M.; Casalini, S.; Berto, M.; Campana, A.; Cramer, T.; Murgia, M.; Geoghegan, M.; Bortolotti, C. A.; Biscarini, F. The Substrate Is a PH-Controlled Second Gate of Electrolyte-Gated Organic Field-Effect Transistor. ACS Appl. Mater. Interfaces 2016, 8, 31783-31790.

(10) Di Lauro, M.; Berto, M.; Giordani, M.; Benaglia, S.; Schweicher, G.; Vuillaume, D.; Bortolotti, C. A.; Geerts, Y. H.; Biscarini, F. Liquid-Gated Organic Electronic Devices Based on HighPerformance Solution-Processed Molecular Semiconductor. Adv. Electron. Mater. 2017, 3, No. 1700159.

(11) Tarabella, G.; Santato, C.; Yang, S. Y.; Iannotta, S.; Malliaras, G. G.; Cicoira, F. Effect of the Gate Electrode on the Response of Organic Electrochemical Transistors. Appl. Phys. Lett. 2010, 97, No. 123304.

(12) Rivnay, J.; Inal, S.; Collins, B. A.; Sessolo, M.; Stavrinidou, E.; Strakosas, X.; Tassone, C.; Delongchamp, D. M.; Malliaras, G. G. Structural Control of Mixed Ionic and Electronic Transport in Conducting Polymers. Nat. Commun. 2016, 7, No. 11287.

(13) Lago, N.; Cester, A.; Wrachien, N.; Natali, M.; Quiroga, S. D.; Bonetti, S.; Barbato, M.; Rizzo, A.; Benvenuti, E.; Benfenati, V.; et al. A Physical-Based Equivalent Circuit Model for an Organic/Electrolyte Interface. Org. Electron. 2016, 35, 176-185.
(14) van de Burgt, Y.; Melianas, A.; Keene, S. T.; Malliaras, G.; Salleo, A. Organic Electronics for Neuromorphic Computing. Nat. Electron. 2018, 1, 386-397.

(15) Someya, T.; Bao, Z.; Malliaras, G. G. The Rise of Plastic Bioelectronics. Nature 2016, 540, 379-385.

(16) Berto, M.; Diacci, C.; Theuer, L.; Di Lauro, M.; Simon, D. T.; Berggren, M.; Biscarini, F.; Beni, V.; Bortolotti, C. A. Label Free Urea Biosensor Based on Organic Electrochemical Transistors. Flex. Print. Electron. 2018, 3, No. 024001.

(17) Berggren, M.; Nilsson, D.; Robinson, N. D. Organic Materials for Printed Electronics. Nat. Mater. 2007, 6, 3-5.

(18) Casalini, S.; Bortolotti, C. A.; Leonardi, F.; Biscarini, F. SelfAssembled Monolayers in Organic Electronics. Chem. Soc. Rev. 2017, $46,40-71$.

(19) Schmaltz, T.; Sforazzini, G.; Reichert, T.; Frauenrath, H. SelfAssembled Monolayers as Patterning Tool for Organic Electronic Devices. Adv. Mater. 2017, 29, No. 1605286.

(20) White, S. P.; Dorfman, K. D.; Frisbie, C. D. Operating and Sensing Mechanism of Electrolyte-Gated Transistors with Floating Gates: Building a Platform for Amplified Biodetection. J. Phys. Chem. C 2016, 120, 108-117.

(21) Macchia, E.; Manoli, K.; Holzer, B.; Di Franco, C.; Ghittorelli, M.; Torricelli, F.; Alberga, D.; Mangiatordi, G. F.; Palazzo, G.; Scamarcio, G.; et al. Single-Molecule Detection with a MillimetreSized Transistor. Nat. Commun. 2018, 9, No. 3223.

(22) Casalini, S.; Dumitru, A. C.; Leonardi, F.; Bortolotti, C. A.; Herruzo, E. T.; Campana, A.; De Oliveira, R. F.; Cramer, T.; Garcia, R.; Biscarini, F. Multiscale Sensing of Antibody-Antigen Interactions by Organic Transistors and Single-Molecule Force Spectroscopy. ACS Nano 2015, 9, 5051-5062.

(23) Ma, F.; Lennox, R. B. Potential-Assisted Deposition of Alkanethiols on Au: Controlled Preparation of Single- and MixedComponent SAMs. Langmuir 2000, 16, 6188-6190.

(24) Haag, A. L.; Toader, V.; Lennox, R. B.; Grutter, P. Selective in Situ Potential-Assisted SAM Formation on Multi Electrode Arrays. Nanotechnology 2016, 27, No. 455501.

(25) Jambrec, D.; Gebala, M.; La Mantia, F.; Schuhmann, W. Potential-Assisted DNA Immobilization as a Prerequisite for Fast and Controlled Formation of DNA Monolayers. Angew. Chem., Int. Ed. 2015, 54, 15064-15068.

(26) Lin, F.; Lonergan, M. C. Gate Electrode Processes in an Electrolyte-Gated Transistor: Non-Faradaically versus Faradaically Coupled Conductivity Modulation of a Polyacetylene Ionomer. Appl. Phys. Lett. 2006, 88, No. 133507.

(27) Demelas, M.; Scavetta, E.; Basiricò, L.; Rogani, R.; Bonfiglio, A. A Deeper Insight into the Operation Regime of All-Polymeric Electrochemical Transistors. Appl. Phys. Lett. 2013, 102, No. 193301.

(28) Bernards, D. A.; MacAya, D. J.; Nikolou, M.; Defranco, J. A.; Takamatsu, S.; Malliaras, G. G. Enzymatic Sensing with Organic Electrochemical Transistors. J. Mater. Chem. 2008, 18, 116-120.

(29) Tang, H.; Lin, P.; Chan, H. L. W.; Yan, F. Highly Sensitive Dopamine Biosensors Based on Organic Electrochemical Transistors. Biosens. Bioelectron. 2011, 26, 4559-4563.

(30) Wang, F.; Zhang, Y.; Liang, W.; Chen, L.; Li, Y.; He, X. NonEnzymatic Glucose Sensor with High Sensitivity Based on $\mathrm{Cu}-\mathrm{Al}$ Layered Double Hydroxides. Sens. Actuators, B 2018, 273, 41-47.

(31) Lin, P.; Luo, X.; Hsing, I. M.; Yan, F. Organic Electrochemical Transistors Integrated in Flexible Microfluidic Systems and Used for Label-Free DNA Sensing. Adv. Mater. 2011, 23, 4035-4040.

(32) Spain, E.; Keyes, T. E.; Forster, R. J. DNA Sensor Based on Vapour Polymerised Pedot Films Functionalised with Gold Nanoparticles. Biosens. Bioelectron. 2013, 41, 65-70.

(33) Uda, M.; Nakamura, A.; Yamamoto, T.; Fujimoto, Y. Work Function of Polycrystalline Ag, Au and Al. J. Electron. Spectrosc. Relat. Phenom. 1998, 88-91, 643-648.

(34) Beni, V.; Newton, H. V.; Arrigan, D. W. M.; Hill, M.; Lane, W. A.; Mathewson, A. Voltammetric Behaviour at Gold Electrodes Immersed in the BCR Sequential Extraction Scheme Media: Application of Underpotential Deposition-Stripping Voltammetry to 
Determination of Copper in Soil Extracts. Anal. Chim. Acta 2004, 502, 195-206.

(35) Macchia, E.; Romele, P.; Manoli, K.; Ghittorelli, M.; Magliulo, M.; Kovács-Vajna, Z. M.; Torricelli, F.; Torsi, L. Ultra-Sensitive Protein Detection with Organic Electrochemical Transistors Printed on Plastic Substrates. Flexible Printed Electron. 2018, 3, No. 034002.

(36) Casalini, S.; Berto, M.; Kovtun, A.; Operamolla, A.; Di Rocco, G.; Facci, P.; Liscio, A.; Farinola, G. M.; Borsari, M.; Bortolotti, C. A. Surface Immobilized His-Tagged Azurin as a Model Interface for the Investigation of Vectorial Electron Transfer in Biological Systems. Electrochim. Acta 2015, 178, 638-646.

(37) Jambrec, D.; Conzuelo, F.; Estrada-Vargas, A.; Schuhmann, W. Potential-Pulse-Assisted Formation of Thiol Monolayers within Minutes for Fast and Controlled Electrode Surface Modification. ChemElectroChem 2016, 3, 1484-1489.

(38) Alloway, D. M.; Graham, A. L.; Yang, X.; Mudalige, A.; Colorado, R.; Wysocki, V. H.; Pemberton, J. E.; Lee, T. R.; Wysocki, R. J.; Armstrong, N. R. Tuning the Effective Work Function of Gold and Silver Using -Functionalized Alkanethiols: Varying Surface Composition through Dilution and Choice of Terminal Groups. J. Phys. Chem. C 2009, 113, 20328-20334.

(39) Beni, V.; Gelaw, T. K.; O'Sullivan, C. K. Study of the Combination of the Deposition/Stripping of Sacrificial Metal NanoStructures and Alkanethiol as a Route for Genosensor Surface Preparation. Electrochem. Commun. 2011, 13, 325-327.

(40) Shim, N. Y.; Bernards, D. A.; Macaya, D. J.; DeFranco, J. A.; Nikolou, M.; Owens, R. M.; Malliaras, G. G. All-Plastic Electrochemical Transistor for Glucose Sensing Using a Ferrocene Mediator. Sensors 2009, 9, 9896-9902.

(41) Bortolotti, C. A.; Paltrinieri, L.; Monari, S.; Ranieri, A.; Borsari, M.; Battistuzzi, G.; Sola, M. A Surface-Immobilized Cytochrome c Variant Provides a PH-Controlled Molecular Switch. Chem. Sci. 2012, 3, 807-810.

(42) Liscio, A. Scanning Probe Microscopy beyond Imaging: A General Tool for Quantitative Analysis. ChemPhysChem 2013, 14, 1283-1292.

(43) Liscio, A.; Palermo, V.; Samorì, P. Nanoscale Quantitative Measurement of the Potential of Charged Nanostructures by Electrostatic and Kelvin Probe Force Microscopy: Unraveling Electronic Processes in Complex Materials. Acc. Chem. Res. 2010, 43, 541-550.

(44) Musumeci, C.; Liscio, A.; Palermo, V.; Samorì, P. Electronic Characterization of Supramolecular Materials at the Nanoscale by Conductive Atomic Force and Kelvin Probe Force Microscopies. Mater. Today 2014, 17, 504-517.

(45) Hansen, W. N.; Hansen, G. J. Standard Reference Surfaces for Work Function Measurements in Air. Surf. Sci. 2001, 481, 172-184. 